\title{
Assessment of hair surface roughness by objective analysis
}

\section{Ke un Hyung Park, Hyun Joo Kim, Bobae Oh, Eunyoung Le e, a nd Ja e hyoun Ha}

Skin Re se a re h Institute, IEC Ko re a, Suwon 443-813, Ko re a

\section{Introduction}

There have been growing interests of beauty treatment and skin care around the world. Among these interests, hair care markets are rising rapidly. As a result, various types of hair products are launched and the importance of hair care is issued socially. The keen attention on hair and cuticle is clearly drawn on the rise. Cuticle layer is the firstly exposed area from damage and the region where primary protection occurs. For such reasons, hair products regard the cuticle protection as important. However, previous studies only have provided the results based on visual assessment. Therefore, quantitative evaluation method of hair products is needed. The aims of this study are to obtain the changes of cuticle in image and to measure the hair surface roughness with quantitative image analysis by HIROX.

\section{Materials and Methods}

\section{Subjects and hair samples}

Twenty three female Korean volunteers (mean age, 39.9 years; range, 28-52 years) who considered their hair damaged participated in this 4 week study. Three hairs on the top of the head were collected from each subject on the study start day (D0) before the product use. After effective commercial hair care products were supplied to the subjects, they were informed to use the products for 4 weeks. Three hairs from the same region of the head were collected from each subject after 4 weeks of product use (D28).

\section{Digital microscope image analysis}

For this study, the most recent three dimensional (3D) digital microscope system (KH-8700; HIROX) was used to capture enlarged images of the hair cuticle region. MG(G)-10C:OL-350(II) (HIROX) lenses were used. After setting up the hair sample's lowest and highest focus points, 3D image automatic filming was started. After filming, the images were rendered by the image analysis software, and the visual changes between the hair samples were compared. 3D images for analysis were retrieved from the image analysis software. The roughness parameter was graded as follows: $\mathrm{Ra}$, mean arithmetic roughness; Rz, maximum roughness; and Rzjis, mean roughness of randomly assigned points (based on International Standard Japan Industrial Standard 2001). Before proceeding with the analysis, three longer regions were identified on the hair sample. As a result, 18 roughness parameter values were obtained from each subject on D0 and D28. The nine parameter values provided on each of the two days were expressed as average mean \pm standard deviation.

\section{Results}
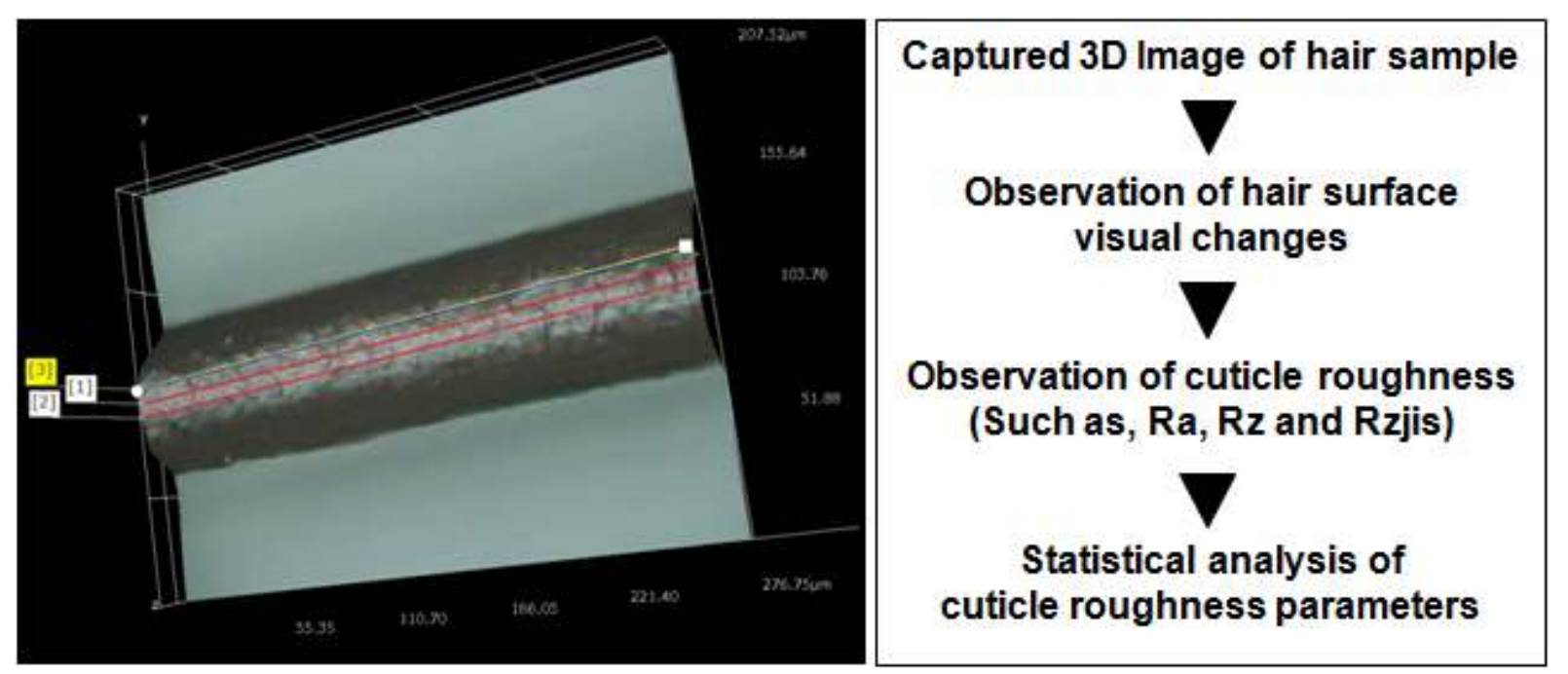

Fig. 1. Sample analysis of hair surface (cuticle) changes using the visual and quantitative methods of digital microscope image analysis.
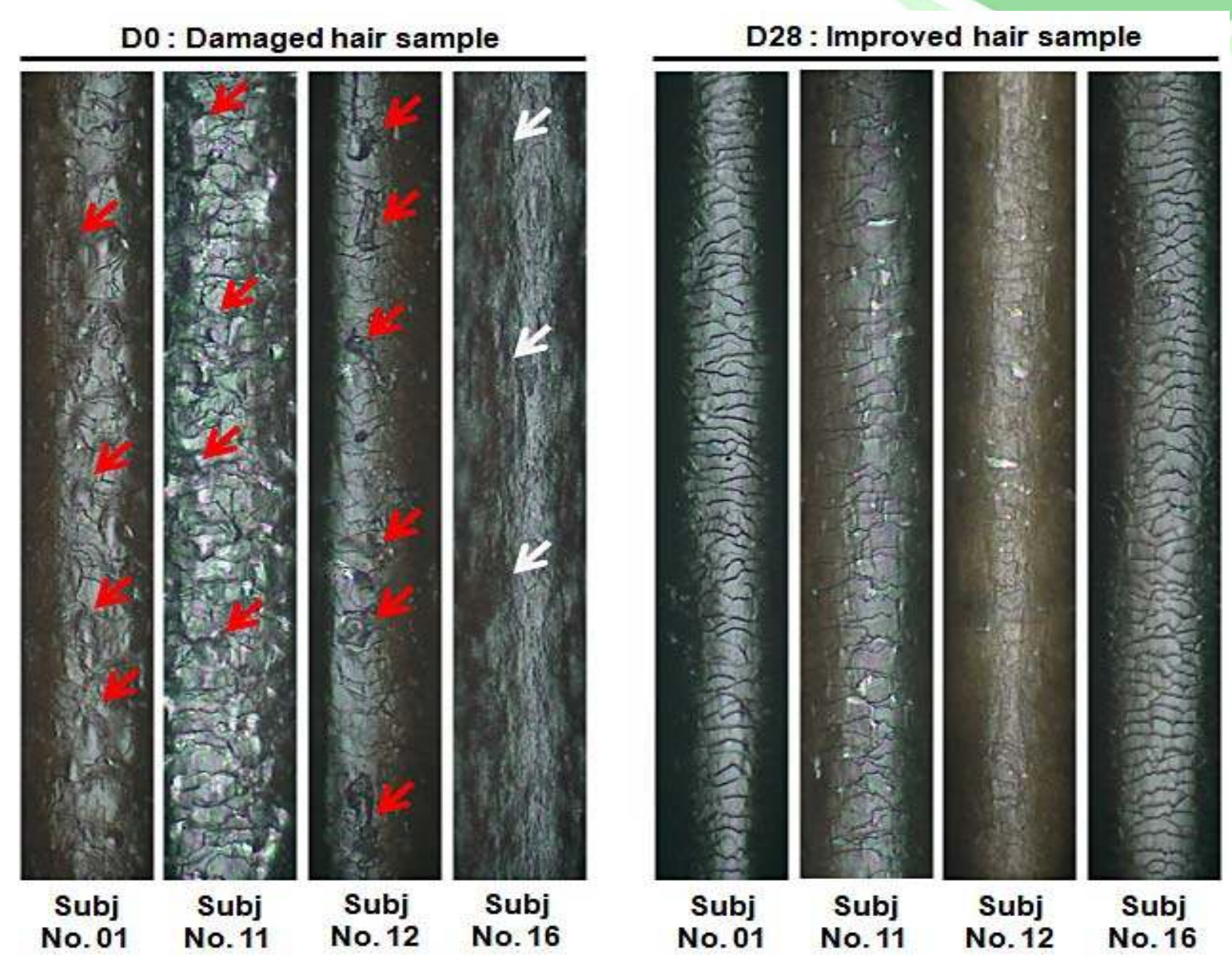

Fig. 2. Comparison of the visual changes on observation of hair surface between damaged hair samples (D0, untreated with the hair care product) and improved hair samples (D28, treated with the hair care product). The red arrows indicate the damaged hair surface (overall severe desquamation of the cuticle), and the white arrows show the hair surface with an exposed cortex and no cuticle (most severe grade). All images are magnified $\times 700$.
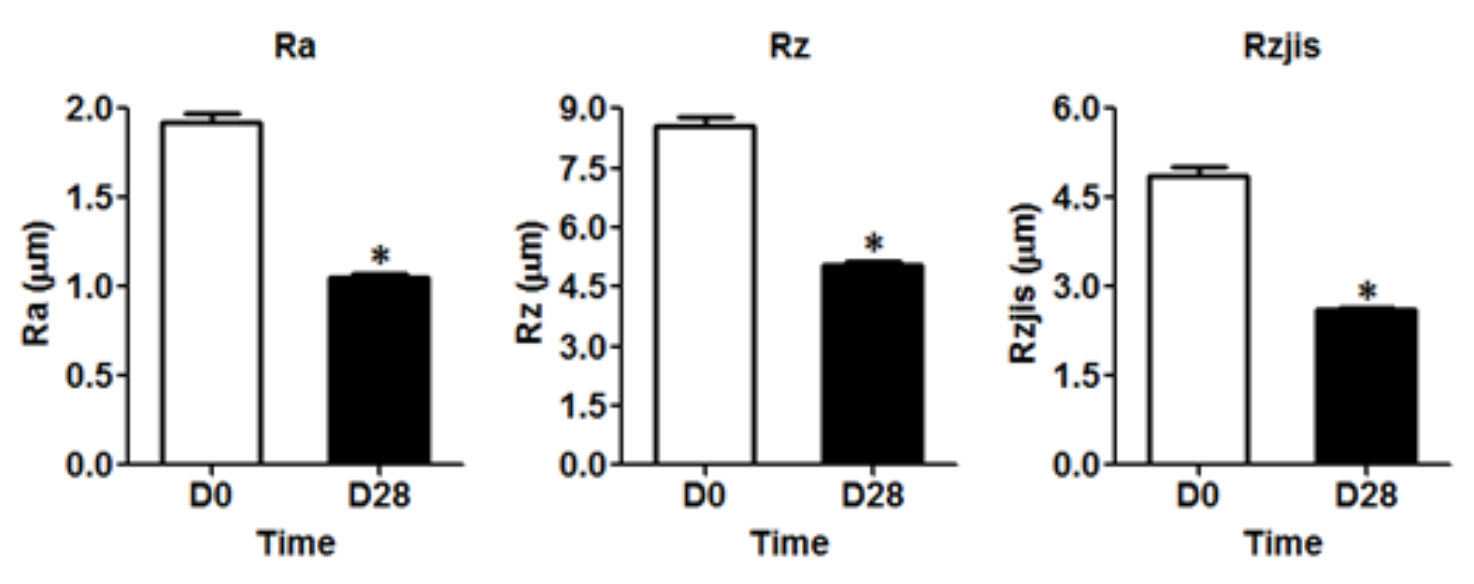

\begin{tabular}{|c|c|c|}
\hline & D0 & D28 \\
\hline Ra $(\mu \mathrm{m})$ & $1.926 \pm 0.237$ & $1.056 \pm 0.066$ \\
$p$-value & $/$ & $<0.001$ \\
\hline $\mathrm{Rz}(\mu \mathrm{m})$ & $8.535 \pm 1.093$ & $5.043 \pm 0.384$ \\
$p$-value & $/$ & $<0.001$ \\
\hline Rzjis $(\mu \mathrm{m})$ & $4.877 \pm 0.623$ & $2.608 \pm 0.183$ \\
$p$-value & $/$ & $<0.001$ \\
\hline
\end{tabular}

Fig. 3. Comparison of quantifiable changes in hair surface (cuticle) parameters between damaged hair samples (D0, untreated with the hair care product) and improved hair samples (D28, treated with the hair care product). Results shown are the mean values of nine independent areas \pm SD. ${ }^{*} p<0.05$ versus damaged hair samples (D0, untreated with hair care products).

\section{Conclusions}

In conclusion, this study showed for the first time that the 3D digital microscope system and software can be used to observe hair cuticle changes and verify quantitative changes using the international standard roughness parameters ( $R a, R z$, and $R z j i s)$. The analysis method in this study will be used to evaluate the effects of hair care products and assess their medical improvements.

\section{References}

1. Lshikawa K, Okamoto M, Aoyagi S. Structural analysis of the outermost hair surface using TOF-SIMS with gas cluster ion beam sputtering. Biointerphases 2016;11:02A315.

2. Park KH, Kim HJ, Oh B, Lee E, Ha J. Assessment of hair surface roughness using quantitative image analysis. Skin Res Technol. 2017 Jul 19. doi: 10.1111/srt.12393. 